\title{
Risk of ambulatory colonoscopy in patients with cirrhosis: a propensity-score matched cohort study
}

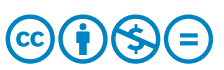

\author{
Authors \\ Robert J. Huang ${ }^{1}$, Subhas Banerjee ${ }^{1}$, Shai Friedland ${ }^{1,2}$, Uri Ladabaum ${ }^{1}$ \\ Institutions \\ 1 Division of Gastroenterology and Hepatology, Stanford \\ University, Stanford, California, United States \\ 2 The Veterans Affairs Palo Alto Health Care System, Palo \\ Alto, California, United States
}

submitted 30.4.2020

accepted after revision 31.7.2020

Bibliography

Endoscopy International Open 2020; 08: E1495-E1501

DOI 10.1055/a-1242-9958

ISSN 2364-3722

(c) 2020. The Author(s).

This is an open access article published by Thieme under the terms of the Creative Commons Attribution-NonDerivative-NonCommercial License, permitting copying and reproduction so long as the original work is given appropriate credit. Contents may not be used for commecial purposes, or adapted, remixed, transformed or built upon. (https://creativecommons.org/licenses/by-nc-nd/4.0/)

\section{Corresponding author}

Robert Huang, MD, MS, Division of Gastroenterology and Hepatology, Stanford University, 300 Pasteur Drive, Alway Building, M211, Stanford, CA 94305, United States

Fax: +1-650-498-6205

rjhuang@stanford.edu

Supplementary material is available under https://doi.org/10.1055/a-1242-9958

\section{ABSTRACT}

Background and study aims Patients with cirrhosis demonstrate alterations in physiology, hemodynamics, and immunity which may increase procedural risk. There exist sparse data regarding the safety of performing ambulatory colonoscopy in patients with cirrhosis.

Patients and methods From a population-based sample of three North American states (California, Florida, and New York), we collected data on 3,590 patients with cirrhosis who underwent ambulatory colonoscopy from 2009 to 2014. We created a control cohort propensity score-matched for cirrhotic severity who did not undergo colonoscopy $(\mathrm{N}=3,590)$ in order to calculate the attributable risk for adverse events. The primary endpoint was the rate of unplanned hospital encounters (UHEs) within 14 days of colonoscopy (or from a synthetic index date for the control cohort). Predictors for UHE were assessed in multivariable regression.

Results The attributable risk for any UHE following colonoscopy was $3.1 \%$ (confidence interval $[\mathrm{Cl}] 2.1-4.1 \%, P<$ $0.001)$. There was increased risk for infection $(0.9 \%, \mathrm{Cl}$ $0.7-1.1 \%)$, spontaneous bacterial peritonitis $(0.1 \%, \mathrm{Cl}$ $0.0-0.3 \%)$, decompensation of ascites $(0.3 \%, \mathrm{Cl} 0.2-$ $0.4 \%)$, and cardiovascular event $(0.4 \%, \mathrm{Cl} 0.3-0.5 \%)$. There was no increased attributable risk for gastrointestinal bleeding, perforation, or development of the hepatorenal syndrome. The presence of ascites at time of procedure was the only predictor for UHE in the fully-adjusted model (OR 2.6, CI 1.9-3.5, $P<0.001$ ).

Conclusions There is a moderate though detectable increase in risk for adverse event following ambulatory colonoscopy in patients with cirrhosis. The presence of ascites in particular portends higher risk. These data may guide clinicians when counseling patients with cirrhosis on the choice of colorectal cancer screening modality.

\section{Introduction}

It is estimated that over 600,000 people live with cirrhosis in North America, with potentially many more patients going undiagnosed [1]. With recent treatments against viral hepatitides, earlier recognition of cirrhosis, and better access to liver trans- plantation, life expectancy for patients with cirrhosis has improved $[1,2]$. An increasing number of patients with cirrhosis may benefit from health maintenance interventions, including colorectal cancer screening $[3,4]$. Higher rates of colorectal neoplasia have been described among patients awaiting liver transplant compared to screening controls $[5,6]$, further em- 
phasizing the importance of appropriate colorectal cancer screening in this group. In the United States, colonoscopy is the most common test performed for colorectal cancer screening [7].

Cirrhosis leads to alterations in physiology, hemodynamics $[8,9]$, immunity $[10,11]$, and coagulation $[12,13]$ which may make colonoscopy both more technically challenging and potentially with greater risk for adverse event. While there has been extensive literature regarding the increased surgical risk faced by patients with cirrhosis[14-18], there exist very limited data regarding risk of colonoscopy in cirrhosis. Two retrospective, single-center studies from South Korea analyzing risk for post-polypectomy bleeding found no increased risk among patients with early cirrhosis [19], but an increased risk among patients with more advanced cirrhosis (Child-Pugh class B or C) [20]. A single-center American study analyzing bleeding also found more advanced cirrhotic features (ascites and the presence of esophageal varices) to predict post-polypectomy bleeding, but with overall low rates of major complication or hospitalization [21]. While there have been case reports of the development of peritonitis following colonoscopy among patients with cirrhosis [22-24], systematic data regarding the risk for infectious complications are lacking. There exist only limited data regarding the potential for colonoscopy, and its associated sedation, to incite liver decompensating events such as spontaneous bacterial peritonitis and the hepatorenal syndrome.

In this population-based study incorporating data from three large American states, we compared the rates of adverse events (AEs) following ambulatory colonoscopy in patients with cirrhosis against a propensity-score matched cirrhotic control cohort, and analyzed risk factors for adverse events.

\section{Patients and methods}

\section{Data sources}

This study was designed as a retrospective cohort study using the state ambulatory surgery, inpatient, and emergency department databases of the U.S. Healthcare Cost and Utilization Project (HCUP) [21]. These databases are compiled from legally-mandated encounter records provided by each licensed facility to state health departments. The states of California (CA), Florida (FL), and New York (NY) are the three largest states that provide longitudinal patient identifiers by which to track subsequent emergency department and hospital encounters. The years 2009 to 2011 for CA, 2012 to 2014 for FL, and 2011 to 2013 for NY were chosen as these were the three most recent years of available data at the time of this study. These three states assign encrypted, individual-level identifiers which remain constant between the three data sets (ambulatory surgery, inpatient, and emergency department) allowing for linkage of patients with subsequent inpatient/emergency department visits following ambulatory procedures.

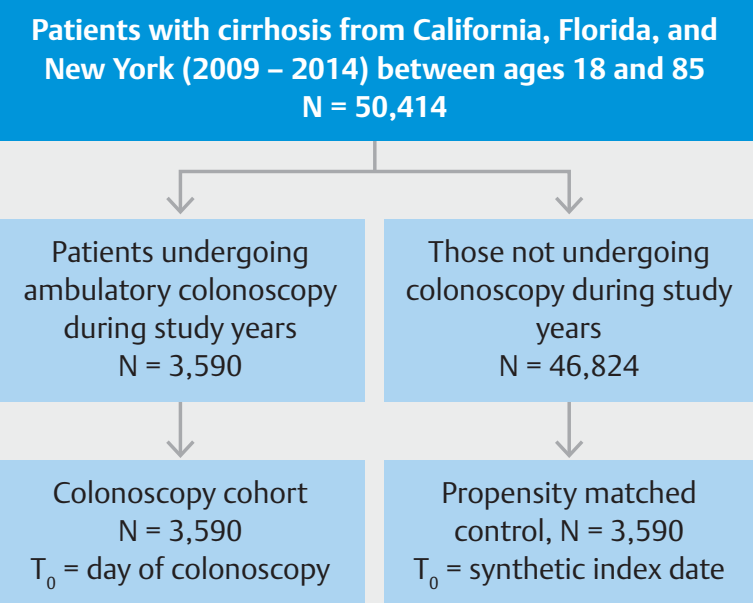

- Fig. 1 Flow diagram of cohort selection and assignment of index date for ascertainment of adverse events.

\section{Cirrhosis cohort}

All patients from the HCUP ambulatory surgery databases from the states of CA, FL, and NY with an ICD-9-CM (International Classification of Diseases, Ninth Revision, Clinical Modification) for cirrhosis $(571.2,571.5)$ were identified from the study years. Of these patients, those younger than 18 or older than 85 years of age were excluded, leaving 50,414 subjects for analysis. From these subjects, those who underwent ambulatory colonoscopy during the study period without concomitant upper endoscopy were identified as the colonoscopy cohort $(\mathrm{N}=$ 3,590 ). From the 46,824 subjects with cirrhosis who did not undergo colonoscopy during the study period, a propensity score (PS) was derived using patient-level factors of age, gender, and presence of cirrhotic sequelae. A PS-matched cohort of 3,590 patients was then created by matching each subject in the colonoscopy cohort with a PS-matched control (with caliper width of 0.0003 , PROC SQL). For the PS-matched controls, a synthetic index date chosen at random from the study period was used as the starting time point $\left(T_{0}\right)$ by which to ascertain development of AEs. A flow diagram demonstrating study design is depicted in > Fig. $\mathbf{1}$.

Patient demographic variables of age, gender, ethnicity/race (defined as white, black, Hispanic [of any race], or other), and primary insurance provider (Medicare, Medicaid, commercial, or uninsured) were recorded. Cirrhosis severity was assessed by identifying the presence of sequelae based upon ICD-9-CM coding (Supplementary Table 1), consisting of a history of esophageal varices, ascites, a history of spontaneous bacterial peritonitis, a history of the hepatorenal syndrome, and total number of cirrhotic sequelae. From the colonoscopy cohort, the performance of biopsy, snare polypectomy, and the indication (diagnostic vs screening/surveillance) were identified based on current procedural terminology codes (Supplementary Table 2). 


\section{Outcome measures}

The main outcome measure was the rate of unplanned hospital encounters (UHEs) within 14 days following colonoscopy or synthetic index date. UHEs included both unplanned admissions as well as emergency department encounters. Unplanned admissions excluded admissions that were coded as scheduled or elective, or whose primary indication was for labor and delivery, maintenance radiation or chemotherapy, or rehabilitation services (Supplementary Table 3) [25]. For the PS-matched control cohort, a randomly-chosen synthetic index date within the study years was assigned to serve as a reference point for the capture of UHEs. Secondary endpoints of the study included UHEs with a principal diagnosis of one of the following events: gastrointestinal bleeding, perforation, infection, cardiovascular event, development of spontaneous bacterial peritonitis, and development of the hepatorenal syndrome. These were recorded based upon ICD-9-CM coding (Supplementary Table4). An additional secondary endpoint was in-hospital mortality within 14 days of colonoscopy or synthetic index date.

\section{Statistical Analysis}

The mean and standard deviation (SD), or the number and percentage of each demographic, clinical, and procedural characteristic were calculated for each cohort. Differences in baseline characteristics were calculated with either Student's $t$-test for continuous variables, or the chi-squared test for categorical variables. The number and percentage of UHEs in aggregate, UHEs with specific AEs, and in-hospital deaths were recorded. The attributable risk of colonoscopy was calculated as the difference in rates of UHE between the colonoscopy and control cohorts [26] To understand risk factors for UHE within the colonoscopy cohort, cirrhotic sequelae were analyzed as predictors in logistic regression using both minimally-adjusted (adjusting for age, gender, and insurance status) and fully-adjusted (with the addition of all cirrhotic sequelae) models. All models estimated the odds ratio (OR) and 95\% confidence intervals $(\mathrm{CI})$, with two-sided $P<0.05$ considered as statistically significant. Adjustments for multiple comparisons was performed using Bonferroni's method. To assess the robustness of the regression model, a priori stratified analysis by age ( $\geq 50$, or $<50$ years of age), sex (male or female), and indication (screening or diagnostic) was performed. Additionally, to account for the potential explanatory effects of facility status (ambulatory surgery centers vs hospital outpatient departments), state of residence (California, New York, or Florida), and patient comorbidity on risk for UHE, these variables were included in the multivariable regression model. To evaluate patient comorbidity, we utilized a point classification system of the Elixhauser comorbidity index [27], and stratified patients based on quartile of comorbidity. All analyses were conducted using SAS 9.4 (SAS Institute Inc., Cary, North Carolina, United States).

\section{Results \\ Cirrhosis cohort profile}

The demographic and clinical characteristics of the colonoscopy cohort and the PS-matched cohort are depicted in $>$ Table 1. Both the colonoscopy and PS-matched cohort contained a higher frequency of males, consistent with the epidemiology of chronic liver disease. Both cohorts were well-balanced with regards to age, gender, and insurance status. The colonoscopy cohort demonstrated a lower frequency of non-Hispanic whites compared to the PS-matched cohort. The two cohorts were well-balanced with regards to measures of cirrhosis severity, including history of esophageal varices $(P=0.2)$, presence of ascites $(P=0.4)$, history of hepatorenal syndrome $(P=0.2)$, and the number of cirrhotic sequelae $(P=0.9)$.

\section{Attributable risk of adverse events}

The frequencies of UHE, UHEs with specific AEs, and in-hospital death for each cohort are shown in $>$ Table 2. There was an increased attributable risk for UHE in the colonoscopy compared to control cohort (attributable risk $3.1 \%$, confidence interval [CI] 2.1-4.1\%). With regards to specific AEs, there was increased attributable risk for infection $(0.9 \%, \mathrm{Cl} 0.7-1.1 \%)$, spontaneous bacterial peritonitis $(0.1 \%, \mathrm{Cl} 0.0-0.3 \%)$, decompensation of ascites $(0.3 \%, \mathrm{Cl} 0.2-0.4 \%)$, abdominal pain (1.5\%, $\mathrm{Cl} 0.8-1.5 \%)$, and cardiovascular event $(0.4 \%, \mathrm{Cl} 0.3-$ $0.5 \%)$. There was no increased attributable risk for gastrointestinal bleeding, perforation, or development of the hepatorenal syndrome. There was also no increased risk for in-hospital death following colonoscopy ( $0.3 \%$ vs $0.2 \%, P=0.3)$.

\section{Risk factors for adverse events}

Clinical predictors for UHE following colonoscopy were analyzed in logistic regression (\$ Table 3 ). In a minimally adjusted model (adjusted only for age, gender, and insurance status), the presence of ascites (OR 2.7, CI 2.0 to 3.6), a history of spontaneous bacterial peritonitis (OR 2.6, CI 1.1 to 6.4) and a history of the hepatorenal syndrome (OR 5.1, $\mathrm{Cl} 1.6$ to 16.2) were associated with risk for UHE. Moreover, increasing number of cirrhotic sequelae demonstrated significant association with risk for UHE $(P<0.001)$. However, in the fully-adjusted model (which incorporated all cirrhotic sequelae in addition to age, gender, and insurance status), only the presence of ascites remained a significant predictor (OR 2.6, CI 1.9 to 3.5, P<0.001) for UHE. The overall medical comorbidity (as assessed by Elixhauser Comorbidity) was not predictive of UHE in either minimally- or fullyadjusted model. The state of performance (California, New York, Florida) was not predictive of UHE in either model. Colonoscopy performance in an ambulatory surgery center (as compared to a hospital outpatient department) was not associated with a higher risk for UHE.

When stratified by age ( $\geq 50$, or $<50$ years of age, Supplementary Table 5), sex (male or female, Supplementary Table 6), and indication (screening or diagnostic, Supplementary Table 7), the presence of ascites remained the only significant predictor for UHE following colonoscopy. Similarly, when strati- 
- Table 1 Baseline characteristics of colonoscopy and propensity score-matched control cohorts with regards to demographic and cirrhotic characteristics.

\begin{tabular}{|c|c|c|c|}
\hline \multirow[t]{2}{*}{ Covariate } & \multicolumn{2}{|c|}{ Cohort, Mean (SD) or Frequency (\%) } & \multirow[t]{2}{*}{$P$ value } \\
\hline & Colonoscopy cohort $(\mathrm{N}=3590)$ & Propensity score-matched cohort $(\mathrm{N}=3590)$ & \\
\hline \multicolumn{4}{|l|}{ Demographic Characteristic } \\
\hline - Age & $59.1(9.2)$ & $58.5(10.3)$ & 0.6 \\
\hline - Female & $1,407(39.3 \%)$ & $1,410(39.6 \%)$ & 0.8 \\
\hline \multicolumn{3}{|l|}{ Ethnicity/Race } & $<0.001$ \\
\hline - Non-Hispanic White & $2,147(61.9 \%)$ & $2,237(64.7 \%)$ & \\
\hline - Non-Hispanic Black & $216(6.2 \%)$ & $97(2.8 \%)$ & \\
\hline - Hispanic (any race) & $810(23.3 \%)$ & $529(15.3 \%)$ & \\
\hline - Asian/PI & $115(3.3 \%)$ & $537(15.5 \%)$ & \\
\hline - Other & $183(5.3 \%)$ & $59(1.7 \%)$ & \\
\hline \multicolumn{3}{|l|}{ Primary Insurance } & 0.6 \\
\hline - Medicare & $1,400(39.2 \%)$ & $1,442(40.2 \%)$ & \\
\hline - Medicaid & $819(22.9 \%)$ & $822(22.9 \%)$ & \\
\hline - Commercial & $1,093(30.6 \%)$ & $1,060(29.5 \%)$ & \\
\hline - Uninsured or other & $263(7.6 \%)$ & $266(7.4 \%)$ & \\
\hline \multicolumn{4}{|l|}{ Cirrhosis severity } \\
\hline History of esophageal varices & $255(7.1 \%)$ & $285(7.9 \%)$ & 0.2 \\
\hline Ascites & $646(18.0 \%)$ & $675(18.8 \%)$ & 0.4 \\
\hline History of the hepatorenal syndrome & $15(0.4 \%)$ & $23(0.6 \%)$ & 0.2 \\
\hline \multicolumn{3}{|l|}{ Number of sequelae } & 0.9 \\
\hline .0 & $2,763(77.0 \%)$ & $2,750(76.6 \%)$ & \\
\hline . 1 & $677(18.9 \%)$ & $690(19.2 \%)$ & \\
\hline - 2 or more & $150(4.2 \%)$ & $150(4.2 \%)$ & \\
\hline
\end{tabular}

fied by comorbidity (Supplementary Table 8) and state (Supplementary Table 9), ascites remained the only predictor for UHE. Procedural characteristics were analyzed as risk factors for UHE (Supplementary Table 10). Neither the performance of biopsy nor snare polypectomy predicted UHE in either minimally- or fully-adjusted models. Secondary analysis was performed restricted to UHEs with a principal diagnosis of gastrointestinal bleeding. Neither biopsy nor snare polypectomy predicted risk for UHE with gastrointestinal bleeding.

\section{Discussion}

In this population-level cohort study, we found a significantly increased risk for unplanned hospitalizations and emergency department encounters among patients with cirrhosis undergoing ambulatory colonoscopy compared to matched controls. This risk was most pronounced among patients with ascites at time of colonoscopy. However, there was no increased risk of inhospital death following colonoscopy in patients with cirrhosis.
There has been extensive literature published on the increased surgical risk faced by patients with chronic liver disease [14-18], and scoring systems such as the Child-Pugh score have been developed to specifically estimate perioperative mortality [28-29]. While colonoscopy is performed more than 15 million times annually in the United States [30], there are surprisingly few data regarding endoscopic safety in patients with chronic liver disease. We believe our study is one of the first to offer population-level estimates of endoscopic risk in patients with cirrhosis. Our study suggests that the increased risk for UHE following ambulatory colonoscopy are a result of increased risk for infection (including bacterial peritonitis), cardiovascular events, and decompensated ascites. Immunologic, physiologic, and hemodynamic changes from cirrhosis may all be contributory to these findings.

Multiple components of the systemic immune system are impaired in cirrhosis, including dysregulation of circulating cytokines, decrease in phagocytic activity, and a reduction in serum albumin [10,31,32]. Moreover, hemodynamic changes such as increased portal pressure gradients and the presence 
Table2 Number and frequency of adverse events following ambulatory colonoscopy or synthetic index date (propensity score-matched cohort).

\begin{tabular}{|l|l|l|l|}
\hline Event & Cohort, no. of events (\%) & Attributable risk (Cl) \\
\hline & $\begin{array}{l}\text { Colonoscopy cohort } \\
(\mathbf{N}=\mathbf{3 5 9 )}\end{array}$ & $\begin{array}{l}\text { Propensity score-matched } \\
\text { cohort (N=359) }\end{array}$ & \\
\hline UHE (any cause) & $236(6.6 \%)$ & $124(3.5 \%)$ & $3.1 \%(2.1$ to $4.1 \%)$ \\
\hline Gastrointestinal bleeding & $64(1.8 \%)$ & $82(2.3 \%)$ & $-0.5 \%(-1.1$ to $0.2 \%)$ \\
\hline Perforation & No events & No events & - \\
\hline Infection & $50(1.4 \%)$ & $19(0.5 \%)$ & $0.9 \%(0.7-1.1 \%)$ \\
\hline Spontaneous bacterial peritonitis & $5(0.14 \%)$ & No events & $0.1 \%(0.0$ to $0.3 \%)$ \\
\hline Hepatorenal syndrome & $3(0.08 \%)$ & $2(0.06 \%)$ & $0.0 \%(0.0$ to $0.1 \%)$ \\
\hline Decompensated ascites & $12(0.3 \%)$ & No events & $0.3 \%(0.2-0.4 \%)$ \\
\hline Abdominal pain & $70(2.0 \%)$ & $18(0.5 \%)$ & $1.5 \%(0.8-1.5 \%)$ \\
\hline Cardiovascular event & $15(0.4 \%)$ & No events & $0.4 \%(0.2$ to $0.6 \%)$ \\
\hline UHE from all other causes & $17(0.5 \%)$ & $3(0.008 \%)$ & $0.4 \%(0.3-0.5 \%)$ \\
\hline In-hospital death & $12(0.3 \%)$ & $7(0.2 \%)$ & $0.1 \%(-0.1$ to $0.2 \%)$ \\
\hline UHE, unplanned hospital encounter. & & $<0.001$ \\
\hline Attributable risk defined as difference in rates of UHE (unplanned hospital encounter) between the colonoscopy and control cohort.
\end{tabular}

- Table 3 Logistic regression of unplanned hospital encounter following ambulatory colonoscopy $(N=3590)$.

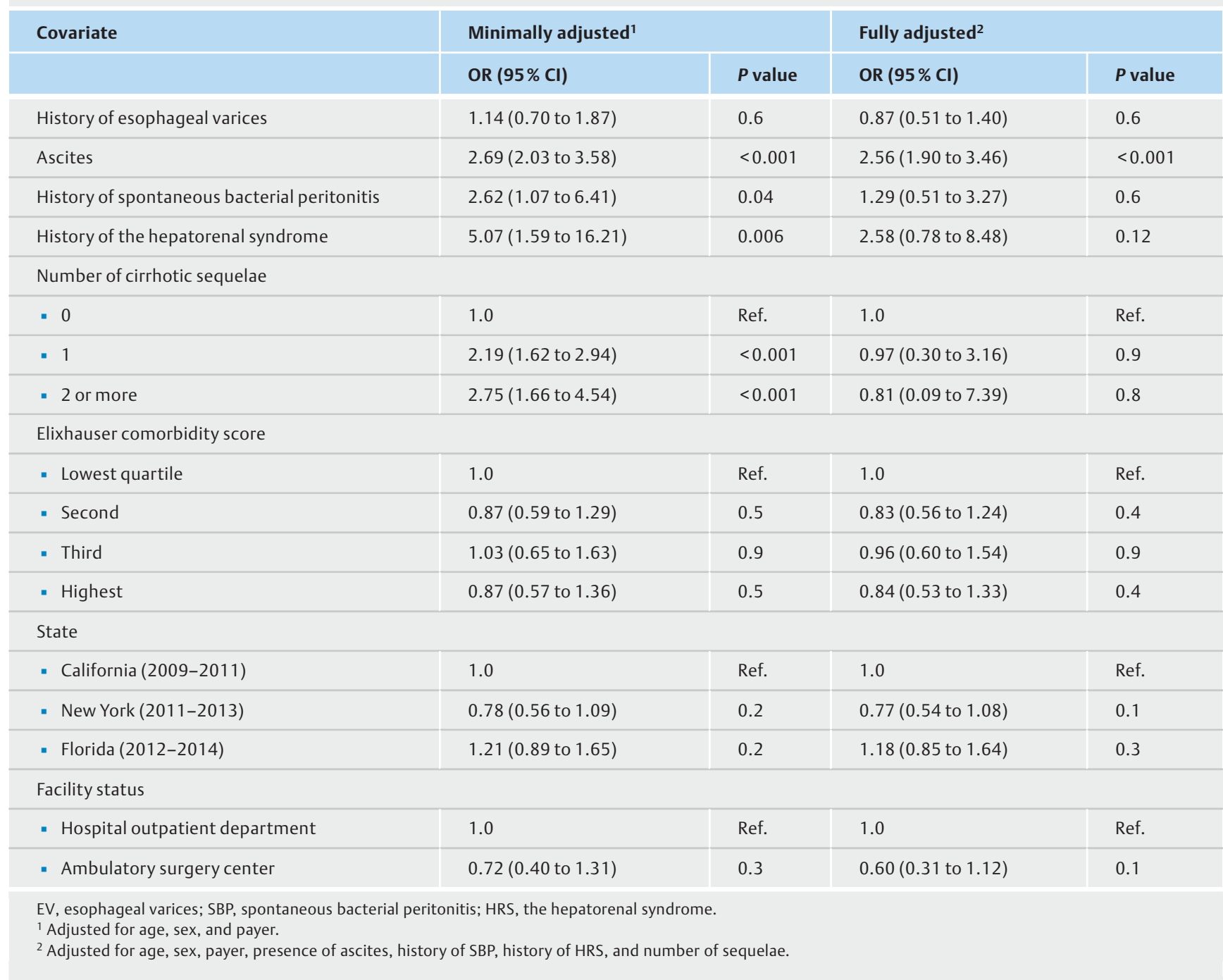


of an aqueous medium (ascites) may also facilitate bacterial translocation and colonization [32,34]. While there have been case reports describing peritonitis following colonoscopy [2224], the rarity of this outcome has made its incidence difficult to study. In this population-level study, we noted five cases of peritonitis within 14 days of colonoscopy. While this incidence is still relatively low ( $0.8 \%$ of patients with ascites), it was significantly higher than among the control cohort (where no episodes occurred). There currently is no recommendation regarding the use of prophylactic antibiotics in patients with cirrhosis undergoing elective colonoscopy, though the American Society of Gastrointestinal Endoscopy does recommend prophylaxis in patients undergoing peritoneal dialysis [35].

With regard to cardiovascular events, the physiologic changes of cirrhosis may alter the clearance of commonly-used anesthetic agents such as benzodiazepines and opiates [36]. Electrolyte derangements and fluid shifts may make patients with cirrhosis more susceptible to arrhythmias and other cardiac events [37]. Moreover, even at baseline patients with cirrhosis have elevated rates of coronary artery disease [38]. However, we observed that baseline general medical comorbidity (as assessed by the Elixhauser index) was not associated with increased risk for post-procedural event. Notably, comorbidity indices (such as Elixhauser and the Charlson Index) include cirrhosis as only a single element among many [39], and degree of cirrhotic severity is not assessed. The results of our study emphasize the need for scoring systems specific to patients with liver disease to establish risk for post-procedural adverse events.

Of the cirrhotic features analyzed, the presence of ascites emerged as the only significant risk factor for adverse events in multivariable analysis. This finding may partially be explained by increased risk for infections including spontaneous bacterial peritonitis. Moreover, the use of diuretics in patients with ascites, frequency of electrolyte disturbances such as hyponatremia [40], and fluid shifts due to fasting or administration of bowel preparation [41] for colonoscopy may all increase risk for cardiovascular events or ascites decompensation in the peri-operative period. Clinicians must be cognizant of this increased risk for decompensation, and exercise caution when referring patients with cirrhosis with ascites for colonoscopy.

Our study has numerous strengths, including the use of a large, population-level cohort with detailed linkage allowing individuals to be followed across multiple facilities and limiting loss to follow-up. There was very little missing information, and granular clinical and procedural covariates could be captured on all individuals. This study used a PS-matched cohort to control for the known increased background risk of hospitalization among patients with cirrhosis $[42,43]$. The matched cohorts were well-balanced with regards to cirrhosis-related sequelae such as the presence of esophageal varices, ascites, and history of decompensating events. Limitations of this study include the possibility of confounding by indication. The use of a PS-matched cohort was an attempt to mitigate this; however, unmeasured confounding may remain as clinicians may refer only healthier patients for colonoscopic evaluation. If so, this effect may actually bias toward the null, and the risk estimates presented in this study may even be underestimated. Laboratory data are not captured in these data sets; therefore, commonly used measures of cirrhotic severity such as the Model for End Stage Liver Disease or Child-Pugh scoring could not be used. Moreover, pharmacy and pathology data are not captured in this study and potential effect modifiers of the association between procedure and outcomes (e.g. aspirin or anticoagulant use) would be missed. Misclassification of outcome is possible, as patients who live close to a state border and who are admitted to a hospital in a neighboring state would not be included for analysis. Only inpatient deaths were captured by the data sets, therefore differences in out-of-hospital mortality could not be assessed between cohorts. Colonoscopies with biopsy or polypectomy may have been performed for screening or surveillance, which cannot be determined based on the procedure codes, but indication was not a significant predictor of outcomes. The data from different states were reported from different years, and there may be secular effects (e.g. improving colonoscopy technology) over the analytic period.

\section{Conclusion}

As diagnosis and management of chronic liver disease continues to improve over the coming years, the number of people living with cirrhosis will only grow. As patients with cirrhosis may be at increased risk for colonic neoplasia [44], physicians and patients must hold informed discussion regarding the relative merits and risks of various colorectal cancer screening modalities. The results of this study will aid clinicians in better understanding the risks and benefits of performing colonoscopy-based evaluation in this group.

\section{Competing interests}

The authors declare that they have no conflict of interest.

References

[1] Scaglione S, Kliethermes S, Cao G et al. The epidemiology of cirrhosis in the United States: a population-based study. J Clin Gastroenterol 2015; 49: 690-696

[2] D'Amico G, Garcia-Tsao G, Pagliaro L. Natural history and prognostic indicators of survival in cirrhosis: a systematic review of 118 studies. J Hepatol 2006; 44: 217-231

[3] Lieberman DA, Rex DK, Winawer S] et al. Guidelines for colonoscopy surveillance after screening and polypectomy: a consensus update by the US Multi-Society Task Force on Colorectal Cancer. Gastroenterology 2012; 143: 844-857

[4] Rex DK, Johnson DA, Anderson JC et al. American College of Gastroenterology guidelines for colorectal cancer screening 2009 [corrected]. Am J Gastroenterol 2009; 104: 739-750

[5] Atassi T, Thuluvath PJ. Risk of colorectal adenoma in liver transplant recipients compared to immunocompetent control population undergoing routine screening colonoscopy. J Clin Gastroenterol 2003; 37: $72-73$ 
[6] Jeschek P, Ferlitsch A, Salzl P et al. A greater proportion of liver transplant candidates have colorectal neoplasia than in the healthy screening population. Clin Gastroenterol Hepatol 2015; 13: 956-962

[7] Sauer AG, Liu B, Siegel RL et al. Comparing cancer screening estimates: Behavioral Risk Factor Surveillance System and National Health Interview Survey. Prev Med 2018; 106: 94-100

[8] Ito K, Shiraki K, Sakai T et al. Portal hypertensive colopathy in patients with liver cirrhosis. World J Gastroenterol 2005; 11: 3127-3130

[9] Runyon BA. Management of Adult Patients with Ascites Due to Cirrhosis: Update 2012. In: Alexandria (VA): American Association for the Study of Liver Diseases. 2013

[10] Sipeki N, Antal-Szalmas P, Lakatos PL et al. Immune dysfunction in cirrhosis. World J Gastroenterol 2014; 20: 2564-2577

[11] Noor MT, Manoria P. Immune dysfunction in cirrhosis. J Clin Trans Hepatol 2017; 5: 50-58

[12] Afdhal N, McHutchison J, Brown R et al. Thrombocytopenia associated with chronic liver disease. J Hepatol 2008; 48: 1000-1007

[13] Tripodi A, Mannucci PM. The coagulopathy of chronic liver disease. N Engl J Med 2011; 365: 147-156

[14] Friedman LS. The risk of surgery in patients with liver disease. Hepatology 1999; 29: 1617-1623

[15] Patel T. Surgery in the patient with liver disease. Mayo Clin Proc 1999; 74: 593-599

[16] O'Leary JG, Yachimski PS, Friedman LS. Surgery in the patient with liver disease. Clin Liver Dis 2009; 13: 211-231

[17] Montomoli ], Erichsen R, Christiansen CF et al. Liver disease and 30day mortality after colorectal cancer surgery: a Danish populationbased cohort study. BMC Gastroenterol 2013; 13: 66

[18] Northup PG, Friedman LS, Kamath PS. AGA Clinical Practice Update on Surgical Risk Assessment and Perioperative Management in Cirrhosis: Expert Review. Clin Gastroenterol Hepatol 2019; 17: 595-606

[19] Jeon JW, Shin HP, Lee Jl et al. The risk of postpolypectomy bleeding during colonoscopy in patients with early liver cirrhosis. Surg Endosc 2012; 26: 3258-3263

[20] Lee S, Park S], Cheon JH et al. Child-Pugh score is an independent risk factor for immediate bleeding after colonoscopic polypectomy in liver cirrhosis. Yonsei Med J 2014; 55: 1281-1288

[21] Huang RJ, Perumpail RB, Thosani N et al. Colonoscopy with polypectomy is associated with a low rate of complications in patients with cirrhosis. Endosc Int Open 2016; 4: E947-952

[22] Christ AD, Bauerfeind P, Gyr N. Peritonitis after colonoscopy in a patient with ascites. Endoscopy 1993; 25: 553-554

[23] Gravito-Soares M, Gravito-Soares E, Lopes S et al. Spontaneous fungal peritonitis: a rare but severe complication of liver cirrhosis. Eur J Gastroenterol Hepatol 2017; 29: 1010-1016

[24] Shrake PD, Troiano F, Rex DK. Peritonitis following colonoscopy in a cirrhotic with ascites. Am J Gastroenterol 1989; 84: 453-454

[25] Ranasinghe I, Parzynski CS, Searfoss R et al. Differences in Colonoscopy quality among facilities: development of a post-colonoscopy risk-standardized rate of unplanned hospital visits. Gastroenterology 2016; 150: 103-113

[26] Rosenberg D, Handler A. Measures of association and hypotheses testing. In: Handler A, Rosenberg D, Monahan C et al. Analytic Methods in Maternal and Child Health.Maternal and Child Health Bureau, HRSA; 1998
[27] van Walraven C, Austin PC, Jennings A et al. A modification of the Elixhauser comorbidity measures into a point system for hospital death using administrative data. Med Care 2009; 47: 626-633

[28] Mansour A, Watson W, Shayani V et al. Abdominal operations in patients with cirrhosis: still a major surgical challenge. Surgery 1997; 122: 730-735; discussion 735-736

[29] Telem DA, Schiano T, Goldstone R et al. Factors that predict outcome of abdominal operations in patients with advanced cirrhosis. Clin Gastroenterol Hepatol 2010; 8: 451-457, quiz e458

[30] Joseph DA, Meester RG, Zauber AG et al. Colorectal cancer screening: Estimated future colonoscopy need and current volume and capacity. Cancer 2016; 122: 2479-2486

[31] Bonnel AR, Bunchorntavakul C, Reddy KR. Immune dysfunction and infections in patients with cirrhosis. Clin Gastroenterol Hepatol 2011; 9: 727-738

[32] Nieto JC, Sanchez E, Romero C et al. Impaired innate immune response of leukocytes from ascitic fluid of patients with spontaneous bacterial peritonitis. J Leukoc Biol 2015; 98: 819-825

[33] Engelmann C, Becker C, Boldt A et al. Ascites' neutrophil function is significantly impaired in patients with decompensated cirrhosis but can be restored by autologous plasma incubation. Sci Rep 2016; 6: 37926

[34] Fagan KJ, Rogers GB, Melino M et al. Ascites bacterial burden and immune cell profile are associated with poor clinical outcomes in the absence of overt infection. PloS One 2015; 10: e0120642

[35] Khashab MA, Chithadi KV. Committee ASoP. et al. Antibiotic prophylaxis for $\mathrm{Gl}$ endoscopy. Gastrointest Endosc 2015; 81: 81-89

[36] Tegeder I, Lotsch J, Geisslinger G. Pharmacokinetics of opioids in liver disease. Clin Pharmacokinet 1999; 37: 17-40

[37] Abbas N, Makker ], Abbas $\mathrm{H}$ et al. Perioperative care of patients with liver cirrhosis: a review. Health Serv Insights 2017; 10: 1178632917691270

[38] Keeling AN, Flaherty JD, Davarpanah AH et al. Coronary multidetector computed tomographic angiography to evaluate coronary artery disease in liver transplant candidates: methods, feasibility and initial experience. J Cardiovasc Med (Hagerstown) 2011; 12: 460-468

[39] Austin SR, Wong YN, Uzzo RG et al. Why summary comorbidity measures such as the Charlson Comorbidity Index and Elixhauser Score Work. Med Care 2015; 53: e65-72

[40] Moore KP, Aithal GP. Guidelines on the management of ascites in cirrhosis. Gut 2006; 55: (Suppl. 06): vi1-12

[41] Clayton DB, Palmer WC, Robison SW et al. Colonoscopy bowel preparation quality improvement for patients with decompensated cirrhosis undergoing evaluation for liver transplantation. Clin Transplant 2016; 30: 1236-1241

[42] Ho CK, Maselli JH, Terrault NA et al. High rate of hospital admissions among patients with cirrhosis seeking care in US emergency departments. Dig Dis Sci 2015; 60: 2183-2189

[43] Volk ML, Tocco RS, Bazick J et al. Hospital readmissions among patients with decompensated cirrhosis. Am J Gastroenterol 2012; 107 : 247-252

[44] Komaki Y, Komaki F, Micic D et al. Risk of colorectal cancer in chronic liver diseases: a systematic review and meta-analysis. Gastrointest Endosc 2017; 86: 93-104 